\title{
A NOTE ON CENTRAL IDEMPOTENTS IN GROUP RINGS II
}

\author{
by SÔNIA P. COELHO and C. POLCINO MILIES
}

(Received 25th June 1986)

Let $G$ be a group and $K$ a field. We shall denote by $U(K G)$ the group of units of the group ring of $G$ over $K$. Also, if $X$ is a group, $T(X)$ will denote the torsion subset of $X$, i.e., the set of all elements of finite order in $X$.

Group theoretical properties of $U(K G)$ have been studied intensively in recent years and it has been found that some conditions about $U(K G)$ imply that $T=T(G)$ must be a subgroup of $G$ and that every idempotent of $K T$ must be central in $K G$.

In a previous note [1], the first author has studied this fact, and determined what it actually implies about the structure of the original group $G$ and the given field $K$, when $\operatorname{char}(K)=p>0$.

Here, we carry on this study to the case where $\operatorname{char}(K)=0$ and prove the following:

Theorem. Let $K$ be a field of characteristic 0 and $T$ the set of torsion elements of a group $G$. Then, every idempotent of $K G$ whose support lies in $T$ is central in $K G$ if and only if:

(i) For every $t \in T$ and every $x \in G$ there exists a positive integer $j$ such that $x t x^{-1}=t^{j}$. Furthermore, for every noncentral element $t \in T, K$ contains no root of unity of order equal to o $(t)$.

(ii) Either $T$ is abelian or $T=A \times E \times K_{8}$ where $A$ is an abelian group such that every element in $A$ is of odd order, $E$ is an elementary abelian 2-group, $K_{8}$ is the quaternion group of order 8 and for every root of unity $\xi$ in an algebraic closure $\Omega$ of $K$ such that $o(\xi)=o(a)$, for some $a \in A$, the field $K(\xi)$ contains no nontrivial solution of the equation $X^{2}+Y^{2}+Z^{2}=0$.

We shall first establish some lemmas.

Lemma 1. If every idempotent $e \in K G$ whose support lies in $T$ is central in $K G$ then we have that:

(i) For every $t \in T$ and every $x \in G$ there exists a positive integer $j$ such that $x t x^{-1}=t^{j}$.

(ii) $T$ is a subgroup of $G$.

This paper was written while the authors were on leave at the University of Alberta, Canada, with partial support from FAPESP and CNPq of Brazil, respectively. 
Proof. Set $t \in T$. Then $e=o(t)^{-1}\left(l+t+\cdots+t^{o(t)-1}\right)$ is an idempotent of $K G$ with $\operatorname{supp}(e) \subset T$, so it is central.

Given any element $x \in G$ we must have that $x e x^{-1}=e$ and, by considering the support of the elements in both sides of this equation, we see that $x t x^{-1}=t^{j}$, for some positive integer $j$.

Now, if $t_{1}, t_{2} \in T$, it is easy to see that $\left(t_{1} t_{2}\right)^{2}=t_{2}^{j} t_{1}^{2} t_{2}$ and, inductively, that $\left(t_{1} t_{2}\right)^{r}=$ $t_{2}^{j} \cdot t_{1}^{r} t_{2}$. So if $m=o\left(t_{1}\right)$ we have that $\left(t_{1} t_{2}\right)^{m} \in\left\langle t_{2}\right\rangle$ is an element of finite order and hence $t_{1} t_{2} \in T$.

Lemma 2. Let $F$ be a field of characteristic $O$. Then $F K_{8}$ contains no noncentral idempotent if and only if the equation $X^{2}+Y^{2}+Z^{2}=0$ has no nontrivial solution in $F$.

Proof. It is well-known that $F K_{8} \cong \mathrm{F} \oplus F \oplus F \oplus F \oplus S$, where $S$ is either a division ring or $S \cong M_{2}(F)$, the ring of $2 \times 2$ matrices with entries in $F$.

Hence, $F K_{8}$ contains a noncentral idempotent if and only if $S \cong M_{2}(F)$, i.e., if and only if $S$ contains a nilpotent element.

Our statement now follows directly from [4, Prop. VI, 1.13].

We conclude our preliminaries with a simple observation. Let $F \subset L$ be fields of characteristic $O$ and $H$ a finite abelian group. Let $\left\{e_{i}\right\}_{1 \leqq i \leqq m}$ and $\left\{f_{j}\right\}_{1 \leqq j \leqq n}$ be the families of idempotents corresponding to the decompositions:

$$
\begin{gathered}
F H=A_{1} \oplus \cdots \oplus A_{m} \\
L H=B_{1} \oplus \cdots \oplus B_{n}
\end{gathered}
$$

of $F H$ and $L H$ as sums of simple components, respectively.

When we extend coefficients from $F H$ to $L H$, each simple component $A_{i}$ of $F H$ splits into a sum of some simple components $\left\{B_{j_{i}}\right\}$ of $L H$. Hence, we have the following:

Remark. Given $e_{r} \neq e_{s}$, we can write $e_{r}=\Sigma f_{j_{r}}$ and $e_{s}=\Sigma f_{j_{s}}$ where both expressions have no commom summand.

We are now ready to prove our main statement.

\section{Proof of the theorem}

Necessity. The first part of condition (i) has been established in Lemma 1. So, let $t$ be a noncentral element in $T$ and let $x \in G$ be such that $x t x^{-1}=t^{j}$ with $j \neq \equiv 1(\bmod o(t))$.

We can write $K\langle t\rangle$ as a sum

$$
K\langle t\rangle \stackrel{\phi}{\cong} K_{1} \oplus \cdots \oplus K_{s}
$$

with $K_{i}=K\left(\xi_{i}\right)$ where $\xi_{i}$ is a root of unity, $1 \leqq i \leqq s$, and at least one of them, $\xi_{1}$ say, is such that $o\left(\xi_{1}\right)=o(t)$.

Here, we have that $\phi(t)=\left(\xi_{1}, \ldots, \xi_{s}\right)$. 
Since every idempotent of $K T$ is central in $K G$, conjugation by $x$ defines an automorphism $\theta: K\langle t\rangle \rightarrow K\langle t\rangle$, which induces automorphisms $\theta_{i}$ on each simple component $K_{i}, 1 \leqq i \leqq s$.

It is easy to see that each $K_{i}$ contains a copy $\bar{K}_{i}$ of $K$, which is fixed under $\theta_{i}$. On the other hand, since $x t x^{-1}=t^{j}$, we see that, in particular, $\theta_{1}\left(\xi_{1}\right)=\xi_{1}^{j} \neq \xi_{1}$ and hence $\xi_{1} \notin K$. This shows that $K$ contains no root of unity of order $o(t)$, as desired.

To establish (ii) we note that Lemma 1 implies that all subgroups in $T$ are normal. Consequently, we know from [2, Theorem 12.5.4] that $T$ is either abelian or a group of the form $T=K_{8} \times A \times E$ as in the statement of our theorem.

So, assume that $T$ is nonabelian and let $a$ be an element in $A$. Once more we write $K\langle a\rangle=K_{1} \oplus \cdots \oplus K_{t}$ with $K_{1}=K\left(\xi_{1}\right)$, where $\xi_{1}$ is a primitive root of unity of order $o\left(\xi_{1}\right)=o(a)$.

Then, the group ring $K\left(\langle a\rangle \times K_{8}\right)$ contains a copy of $K\left(\xi_{1}\right) K_{8}$ and, by hypothesis, every idempotent in $K\left(\xi_{1}\right) K_{8}$ is central. Hence, Lemma 2 shows that the equation $X^{2}+Y^{2}+Z^{2}=0$ has no solution in $K\left(\xi_{1}\right)$.

Sufficiency. Assume first that (i) holds that $T$ is an abelian subgroup of $G$.

Let $e \in K T$ be an idempotent. By considering the subgroup generated by supp (e) we may assume, without loss of generality, that $T$ is finite. Furthermore, since every idempotent of $K T$ is a sum of primitive idempotents we may restrict ourselves to consider the case where $e$ is primitive.

Now, let $\zeta$ be a primitive root of unity of order equal to the exponent of $T$. Then $e \in K T \subset K(\zeta) T$ is a sum of primitive idempotents of $K(\zeta) T$; let $f$ be one of these.

Every $K$-automorphism of $K(\zeta)$ can be extended in a natural way to $K(\zeta) T$. We consider all such automorphisms which give distinct images when computed on $f$ and denote them by $\phi_{1}=I, \phi_{2}, \ldots, \phi_{r}$.

We set $e^{*}=\phi_{1}(f)+\cdots+\phi_{r}(f)$ and we wish to prove that $e^{*}=e$.

Notice that each $\phi_{i}(f), 1 \leqq i \leqq r$, is a primitive idempotent of $K T$. Hence they are pairwise orthogonal and thus $e^{*}=\phi_{1}(f)+\cdots+\phi_{r}(f)$ is also an idempotent.

Since $\phi_{1}(f)$ is a summand in the decompositions of both $e$ and $e^{*}$, due to the remark above it will suffice to show that $e^{*}$ is a primitive idempotent of $K T$.

We shall now prove that the coefficients of $e^{*}$ belong to $K$. Let $\phi$ be any $K$ automorphism of $K(\zeta)$ and denote also by $\phi$ its extension to $K(\zeta) T$.

Then $\phi\left(e^{*}\right)=\sum_{i=1}^{r} \phi \circ \phi_{i}(f)$ and since $\left\{\phi \circ \phi_{i}(f)\right\}_{1 \leqq i \leqq r}=\left\{\phi_{i}(f)\right\}_{1 \leqq i \leqq r}$ it follows that $\phi\left(e^{*}\right)=e^{*}$. Hence, $e^{*} \in K T$.

It remains to show that $e^{*}$ is primitive in $K T$. So, assume that $e^{*}=e_{1}+e_{2}$ where $e_{1}, e_{2} \in K T$ are orthogonal idempotents.

When written as sums of primitive orthogonal idempotents in $K(\zeta) T, e_{1}$ and $e_{2}$ can have no common summand, so, reordering if necessary, we have

$$
e_{1}=\phi_{1}(f)+\cdots+\phi_{l-1}(f) \text { and } e_{2}=\phi_{r}(f)+\cdots+\phi_{r}(f)
$$

Also, since $e_{1} \in K T$, it is fixed by all such $K$-automorphisms, thus, in particular we have:

$$
e_{1}=\phi_{l}\left(e_{1}\right)=\phi_{l} \circ \phi_{1}(f)+\cdots+\phi_{l} \circ \phi_{l-1}(f)
$$


But $\phi_{l} \circ \phi_{1}(f)=\phi_{l}(f)$ so $e_{1}$ and $e_{2}$ would have a common summand, a contradiction.

So, we have established that $e=e^{*}$.

Now, let $x$ be a $n$ arbitrary element in $G$ and write $T=\left\langle t_{1}\right\rangle x \ldots x\left\langle t_{s}\right\rangle$, a direct product of cyclic groups. We can find a positive integer $j$ such that $x t_{k} x^{-1}=t_{k}^{j}$, for every index $k, 1 \leqq k \leqq s$.

According to [3, Theorem 2.12] we can write $f$ in the form:

$$
f=\frac{1}{|T|} \sum_{t \in T} \chi\left(t^{-1}\right) t
$$

where $\chi$ is an irreducible character with values in $K(\zeta)$.

Then:

$$
e=\frac{1}{|T|} \sum_{t \in T}\left(\sum_{i} \phi_{i}\left(\chi\left(t^{-1}\right)\right) t\right.
$$

and hence:

$$
\operatorname{xex}^{-1}=\frac{1}{|T|} \sum_{i \in T}\left(\sum_{i} \phi_{i}\left(\chi\left(t^{-1}\right)\right) t^{j}\right.
$$

So, it will suffice to prove that $\sum_{i} \phi_{i}(\chi(t))=\sum_{i} \phi_{i}\left(\chi\left(t^{j}\right)\right)$, for every $t \in T$.

Since $T$ is abelian and $K(\zeta)$ is a splitting field for $T$, it is easy to see that $\chi(t)$ is a primitive root of unity whose order divides $\exp (T)$. So, we may assume that $\chi(t)=\xi$ where $\xi$ is some power of $\zeta$.

By (i), if $t$ is not central (in which case, there would be nothing to prove) then $\zeta \notin K$ and we can define a $K$-automorphism of $K(\zeta)$ such $\phi(\zeta)=\zeta^{j}$ and extend it to a $K$-automorphism of $K(\zeta) T$ in the usual way, which we still denote by $\phi$.

Now, since $\left\{\phi_{i}\right\}_{1 \leqq i \leqq r}=\left\{\phi_{i} \circ \phi\right\}_{i \leqq 1 \leqq r}$, we have:

$$
\sum_{i} \phi_{i}(\chi(t))=\sum_{i} \phi_{i}(\xi)=\sum_{i} \phi_{i} \circ \phi(\xi)=\sum_{i} \phi_{i}\left(\xi^{j}\right)=\sum_{i} \phi_{i}\left(\chi\left(t^{j}\right)\right)
$$

as we intend to prove.

Finally assume that (i) and (ii) hold and that $T$ is not abelian. As before, we may suppose that $T$ is finite.

Once again, let $\zeta$ be a primitive root of unity, of order $o(\zeta)=\exp (A)$. Then, we can write $K(A \times E)=\oplus_{i} K_{i}$, a direct sum of fields, all of which are contained in $K(\zeta)$.

Hence, $K T=K\left(A \times E \times K_{8}\right) \cong K(A \times E) K_{8} \cong \oplus_{i} K_{i} K_{8}$. By our hypothesis, the equation $X^{2}+Y^{2}+Z^{2}=0$ has no nontrivial solution in any of the fields $K_{i}$, so every idempotent of $K_{i} K_{8}$ is central in $K T$ and our given idempotent $e$ is a sum of central idempotents in some of these components.

To show that $e$ is also central in $K G$ consider an arbitrary element $x \in G$. We wish to show that any idempotent in a given component of the form $K_{i} K_{8}$ commutes with $x$. To see this, denote by $\mu_{i}$ the identity element of $K_{i}$. Notice that each $\mu_{i}$ is an idempotent in $K(A \times E)$ and hence is central in $K G$ because of our earlier argument. Then, the 
idempotents in $K_{i} K_{8}$ are precisely those corresponding to its simple components and can be written explicitly. If we give $K_{8}$ by its presentation:

$$
K_{8}=\left\langle a, b \mid a^{4}=1, a^{2}=b^{2}, b a b^{-1}=a^{3}\right\rangle
$$

these idempotents are:

$$
\begin{aligned}
& e_{1}=\frac{\mu_{i}}{8}\left(1+a+a^{2}+a^{3}+b+a b+a^{2} b+a^{3} b\right) \\
& e_{2}=\frac{\mu_{i}}{8}\left(1+a+a^{2}+a^{3}-b-a b-a^{2} b-a^{3} b\right) \\
& e_{3}=\frac{\mu_{i}}{8}\left(1-a+a^{2}-a^{3}+b-a b+a^{2} b-a^{3} b\right) \\
& e_{4}=\frac{\mu_{i}}{8}\left(1-a+a^{2}-a^{3}-b+a b-a^{2} b+a^{3} b\right) \\
& e_{5}=\frac{\mu_{i}}{8}\left(1-a^{2}\right) .
\end{aligned}
$$

Condition (i) implies that either $x a x^{-1}=a$ or $x a x^{-1}=a^{3}$ and that either $x b x^{-1}=b$ or $x b x^{-1}=a^{2} b$. In all cases, it follows easily that all the idempotents above are central and hence, $e$ itself is also central.

\section{REFERENCES}

1. S. P. Coelho, A note on central idempotents in group rings, Proc. Edinburgh Math. Soc., to appear.

2. M. HALL, The Theory of Groups (Macmillan, New York, 1959).

3. I. M. IsaAcs, Character Theory of Finite Groups (Academic Press, New York, 1976).

4. S. K. Sehgal, Topics in Group Rings (Marcel Dekker, New York, 1979).

Instituto de Matemática e Estatistica

Universidade de São Paulo

Caixa Postal 20.570-Ag. Iguatemi

01000-São PaUlo-Brazil 